\title{
Transient Beam Dynamics in the LBL 2 MV Injector
}

\author{
E. Henestroza, D. P. Grote
}

December 7, 1999

U.S. Department of Energy

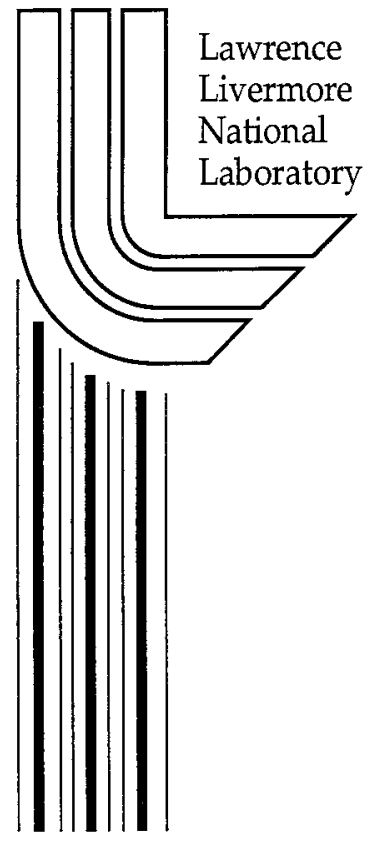




\section{DISCLAIMER}

This document was prepared as an account of work sponsored by an agency of the United States Government. Neither the United States Government nor the University of California nor any of their employees, makes any warranty, express or implied, or assumes any legal liability or responsibility for the accuracy, completeness, or usefulness of any information, apparatus, product, or process disclosed, or represents that its use would not infringe privately owned rights. Reference herein to any specific commercial product, process, or service by trade name, trademark, manufacturer, or otherwise, does not necessarily constitute or imply its endorsement, recommendation, or favoring by the United States Government or the University of California. The views and opinions of authors expressed herein do not necessarily state or reflect those of the United States Government or the University of California, and shall not be used for advertising or product endorsement purposes.

This work was performed under the auspices of the U. S. Department of Energy by the University of California, Lawrence Livermore National Laboratory under Contract No. W-7405-Eng-48.

This report has been reproduced directly from the best available copy.

Available electronically at http://www.doc.gov/bridge

Available for a processing fee to U.S. Department of Energy

And its contractors in paper from

U.S. Department of Energy

Office of Scientific and Technical Information

P.O. Box 62

Oak Ridge, TN 37831-0062

Telephone: (865) 576-8401

Facsimile: (865) 576-5728

E-mail: reports@adonis.osti.gov

Available for the sale to the public from

U.S. Department of Commerce

National Technical Information Service

5285 Port Royal Road

Springfield, VA 22161

Telephone: (800) 553-6847

Facsimile: (703) 605-6900

E-mail: orders@ntis.fedworld.gov

Online ordering: http://www.ntis.gov/ordering.htm

OR

Lawrence Livermore National Laboratory

Technical Information Department's Digital Library

http:/ / www.llnl.gov/tid/Library.html 


\author{
E. Henestroza \\ Lawrence Berkeley National Laboratory, \\ University of California \\ Berkeley, California 94720 \\ D.P. Grote \\ Lawrence Livermore National Laboratory, \\ University of California \\ Livermore, California 94550
}

\section{ABSTRACT}

A driver-scale injector for the Heavy Ion Fusion Accelerator project has been built at LBL. This machine has exceeded the design goals of high voltage ( $>2 \mathrm{MV})$, high current $\left(>0.8 \mathrm{~A}\right.$ of $\left.\mathrm{K}^{+}\right)$and low normalized emittance $(<1 \pi \mathrm{mm}-\mathrm{mr})$. The injector consists of a 750 $\mathrm{keV}$ gun pre-injector followed by an electrostatic quadrupole accelerator (ESQ) which provides strong (alternating gradient) focusing for the space-charge dominated beam, and simultaneously accelerates the ions to $2 \mathrm{MeV}$. A matching section is being built to match the beam to the electrostatic accelerator ELISE. The gun pre-injector, designed to hold up to $1 \mathrm{MV}$ with minimal breakdown risks, consists of a hot aluminosilicate source with a large curved emitting surface surrounded by a thick "extraction electrode." During beam turn-on the voltage at the source is biased from a negative potential, enough to reverse the electric field on the emitting surface and avoid emission, to a positive potential to start extracting the beam; it stays constant for about $1 \mu \mathrm{s}$, and is reversed to turn-off the emission. Since the Marx voltage applied on the accelerating quadrupoles and the main pre-injector gap is a long, constant pulse (several $\mu \mathrm{s}$ ), the transient behavior is dominated by the extraction pulser voltage time profile. The transient longitudinal dynamics of the beam in the injector was simulated by running the Particle in Cell codes GYMNOS and WARP3d in a time dependent mode. The generalization and its implementation in WARP3d of a method proposed by Lampel and Tiefenback to eliminate transient oscillations in a onedimensional planar diode will be presented.

*This work was supported by the Director, Office of Energy Research, Office of Fusion Energy, U.S. Dept. of Energy, under Contract No. DE-AC03-76SF00098 and at LLNL under contract W-7405-ENG-48.

\section{INTRODUCTION}

The design of the $750 \mathrm{keV}$ gun pre-injector, using the gun code EGUN [1], is based on the steadystate space-charge dominated (Child-Langmuir) flow. The relationship between the time-dependent voltage on the "extracting electrode" and the current and energy at the end of the $2 \mathrm{MeV}$ Injector was calculated by the PIC codes GYMNOS[2] and WARP3d[3]. Figure 1 shows current and energy transient oscillations, which decay away within a few multiples of the particle transit time across the gun, for the case of a sudden turn-on of the extracting voltage. The elimination of these oscillations is very important for space-charge dominated beams since such oscillations will lead to envelope variations along the beam which will reduce the dynamic aperture of the accelerator.

This paper discusses the generalization, to any complex geometry, of a method proposed by Lampel and Tiefenback [4] to eliminate transient oscillations in a one-dimensional planar diode.

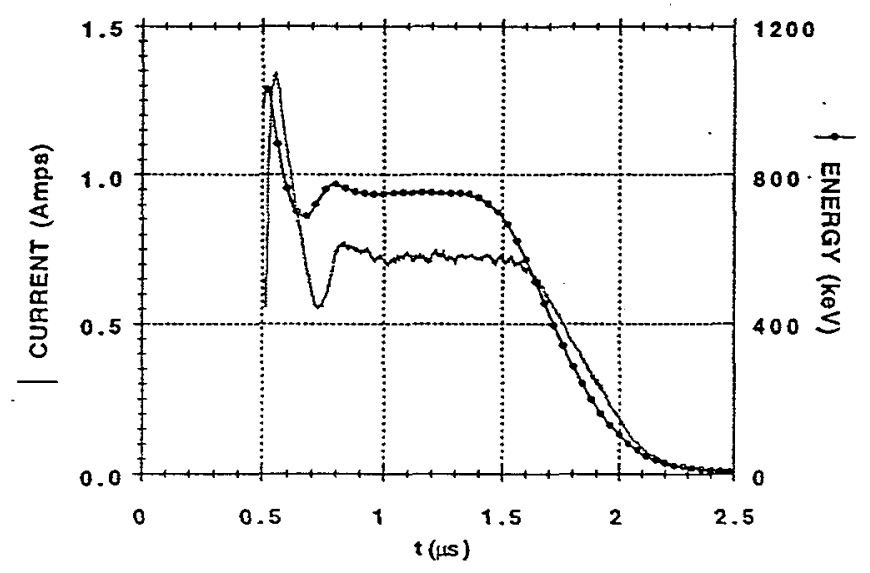

Figure 1: Current and energy transient oscillations for the case of a sudden turn-on of the extracting voltage. 


\section{CURRENT TRANSIENT ELIMINATION IN A 1-D PLANAR DIODE}

In a one-dimensional planar diode it is possible to find an analytic solution for the extracting voltage waveform that will eliminate the current and energy transient oscillations.

Figure 2 shows a schematic diagram of a planar diode. Let $\phi(x)$ be the potential corresponding to the steady-state space-charge dominated flow:

$$
\phi(x)=-V(x / L)^{4 / 3} \text {. }
$$

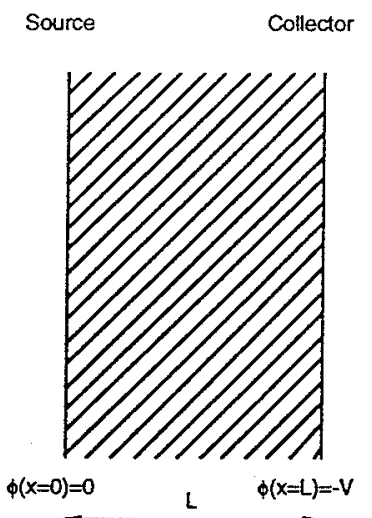

Figure 2: Schematic diagram of a planar diode.

\section{a) Correction of beam head oscillations (Lampel and Tiefenback, 1983).}

To calculate the required $\mathrm{V}(\mathrm{t})$ we use the following procedure starting from the steady-state solution:

- Divide the diode into two regions on either side of the plane at $\mathrm{x}=\xi$.

- Eliminate the charge for $x>\xi$. See Figure 3.

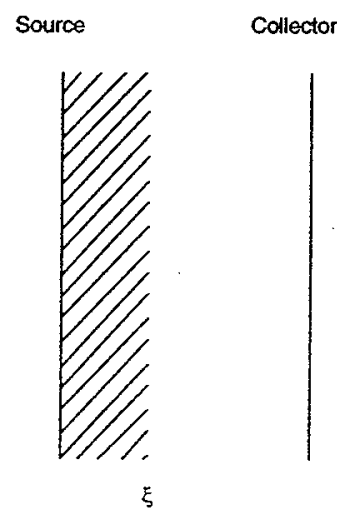

Figure 3: Beam head uniform current flow.

- Keep field solution for $x<\xi$ unchanged by requiring the field at $x=\xi$ to be the same as in the steadystate space-charge dominated flow.
- Change potential at the collector to satisfy Laplace's equation for $x>\xi$, and continuity of potential and field at $x=\xi$. The solution in terms of the parameter $\xi$ is:

$$
\psi_{c}(\xi)=-V\left[4 / 3(\xi / L)^{1 / 3}-1 / 3(\xi / L)^{4 / 3}\right]
$$

- Express the parameter $\xi$ in terms of the time at which the leading particles in the beam are at $\xi$. From energy conservation we get:

$$
1 / 2 \mathrm{~m}(\mathrm{~d} \xi / \mathrm{dt})^{2}=\mathrm{qV}(\xi / L)^{4 / 3}
$$

By integrating the preceding equation we obtain:

$$
\xi / L=(t \tau)^{3}
$$

where the transit time factor is defined as:

$$
\tau=3 \sqrt{ }\left(\mathrm{mL}^{2} /(2 \mathrm{qV})\right)
$$

Since $V(t)=\psi_{c}(\xi)$, the beam-head oscillations will be corrected by applying the following voltage waveform at the collector:

$$
\begin{array}{ll}
V(t)=-V\left[4 / 3(t / \tau)-1 / 3(t / \tau)^{4}\right] & \text { for } t<\tau, \\
V(t)=-V & \text { for } t>\tau .
\end{array}
$$

\section{b) Correction of beam tail oscillations.}

The procedure can be extended to eliminate beam tail oscillations by dividing the diode into two regions on either side of the plane at $x=\xi$, eliminating the charge for $x<\xi$ (see Figure 4), keeping field solution for $x>\xi$ unchanged by requiring the field at $x=\xi$ to be the same as in the steady-state space-charge dominated flow, and changing the potential at the collector to satisfy Laplace's equation for $x<\xi$, and continuity of potential and field at $x=\xi$. The solution in terms of the parameter $\xi$ is:

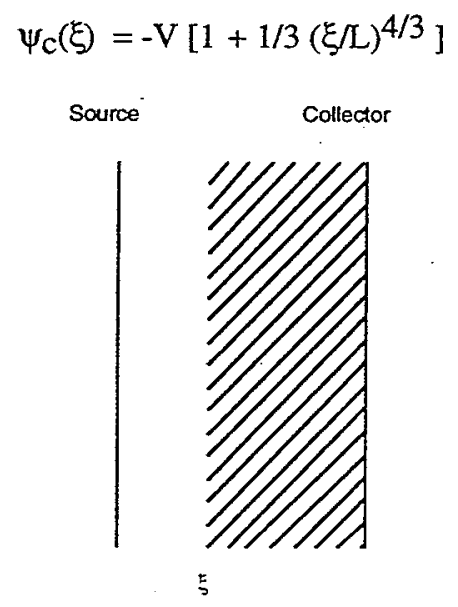

Figure 4: Beam tail uniform current flow.

Taking the time origin as the time when the emission stops, the beam tail oscillations will be corrected by applying the following voltage waveform at the collector:

$$
V(t)=-V\left[1+1 / 3(t / \tau)^{4}\right] \text { for } t>\tau .
$$




\section{CURRENT TRANSIENT ELIMINATION BETWEEN CONCENTRIC SPHERES}

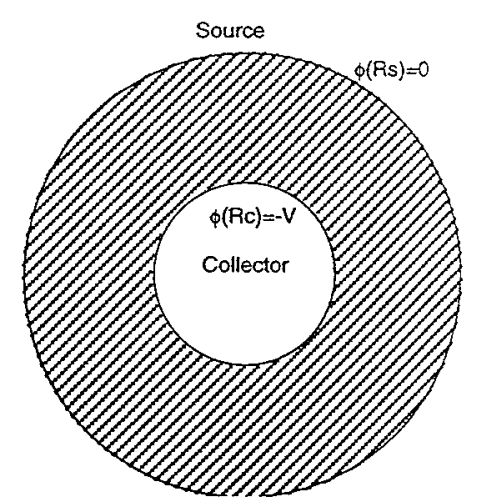

Figure 5: Schematic diagram of a concentric-spheres diode.

For Child-Langmuir flow between concentric spheres (or between infinite coaxial cylinders) the procedure described above provides a set of ordinary differential equations whereby a solution for the extracting voltage wave-form will be obtained that will eliminate the current and energy transient oscillations.

Figure 5 shows a schematic diagram of a diode formed by two concentric spheres. Let $\phi(r)$ be the potential corresponding to the steady-state space-charge dominated flow:

Since

$$
1 / \mathrm{r}^{2} \mathrm{~d} / \mathrm{dr}\left(\mathrm{r}^{2} \mathrm{~d} \phi / \mathrm{dr}\right)=-\rho / \varepsilon 0 .
$$

then

$$
1 / 2 m v^{2}=-q \phi \quad \text { and } \rho=V /\left(4 \pi r^{2} v\right)
$$

$$
\mathrm{d} / \mathrm{dr}\left(\mathrm{r}^{2} \mathrm{~d} \phi / \mathrm{dr}\right)=-\mathrm{I} /\left(4 \pi \varepsilon_{0}\right) \sqrt{ }(-\mathrm{m} /(2 q \phi))
$$

where $v$ and $I$ are the beam velocity and current, respectively.

To correct for beam-head transients we will follow the same procedure described above, starting from the steady-state Child-Langmuir flow solution:

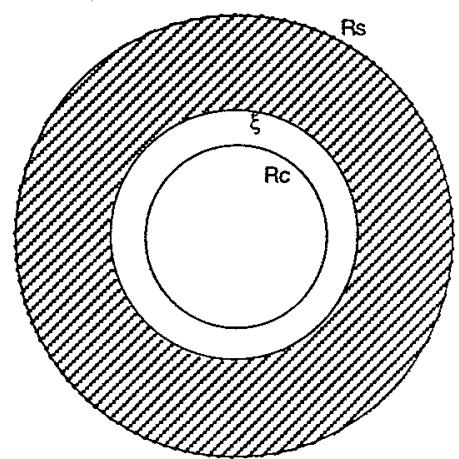

Figure 6: Beam head uniform current flow.
- Divide the diode into two regions on either side of the sphere at $r=\xi$.

- Eliminate the charge for $r<\xi$. See Figure 6 .

- Keep field solution for $r>\xi$ unchanged by requiring the field at $r=\xi$ to be the same as in the steadystate space-charge dominated flow.

- Change potential at the collector to satisfy Laplace's equation for $r<\xi$, and continuity of potential and field at $r=\xi$. The solution in terms of the parameter $\xi$ is:

$$
\psi_{\mathrm{c}}(\xi)=\xi^{2}\left(1 / \xi_{-}-1 / \mathrm{R}_{\mathrm{c}}\right) \phi^{\prime}(\xi)+\phi(\xi)
$$

- Express the parameter $\xi$ in terms of the time at which the leading particles in the beam are at $\xi$. From energy conservation we get (inward flow):

$$
1 / 2 \mathrm{~m}(\mathrm{~d} \xi / \mathrm{dt})^{2}=-\mathrm{q} \phi \text {. }
$$

Since we do not have a closed form solution for $\phi(r)$, then $V(t)$ will be obtained by integrating numerically Poisson's and the velocity equations to obtain $\phi(\xi)$ and $t(\xi)$. The initial conditions are:

$$
\begin{aligned}
& \phi\left(R_{S}\right)=0, \\
& \phi^{\prime}\left(R_{S}\right)=0, \\
& \tau\left(R_{S}\right)=0 .
\end{aligned}
$$

Since $V(t)=\psi_{c}(\xi)$, we obtain the required voltage wave-form by substituting these solutions into the expression for $\psi_{\mathrm{c}}(\xi)$.

A similar analysis, following a different approach, has been reported by Kadish et al. [5].

Particle codes written to study the beam dynamics of one-dimesional diodes have been used to check the validity of the procedure. $[6,7,8]$

\section{CURRENT TRANSIENT ELIMINATION IN GENERAL GEOMETRIES}

The essential feature of the described procedure is to force the field solution in the region occupied by the beam, at any given time, to be the same as the steadystate solution in the same region. This can be done in one-dimensional structures by prescribing an extracting voltage wave-form $V(t)$ since a condition at a single point (the collector) is enough to fulfill the requirement.

For a two- and three-dimensional gun the procedure requires the prescription of extracting voltage waveforms along a curve or on a surface, respectively. Since this requirement is not attainable we can compensate only partially the transient oscillations. For an axisymmetric structure, for example, one could use as an effective potential the average energy of the particles at a given cross section, and follow the same procedure as the one described for one-dimensional structures. 


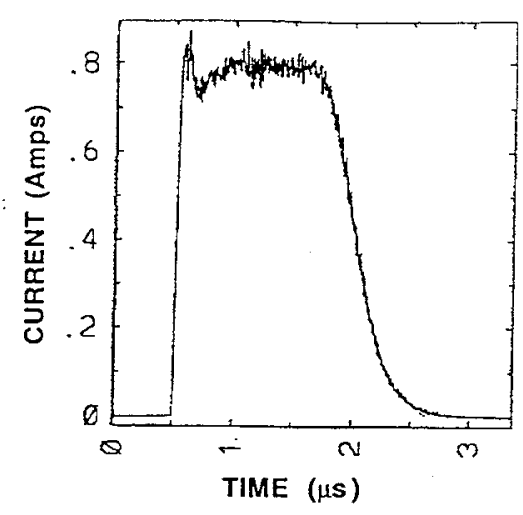

Figure 7: Current profile for the $2 \mathrm{MeV}$ Injector using the numerically-calculated voltage wave-form.

We have implemented the general procedure to eliminate transient oscillations in the three-dimensional PIC code WARP3d. Figure 7 shows the current at the end of the cylindrically symmetric $2 \mathrm{MeV}$ Injector using the numerically calculated voltage wave-form $V(t)$ applied on the extracting electrode. The resulting current profile shows a decrease in the beam-head transient oscillation. Since the $2 \mathrm{MeV}$ HIF Injector Gun is poorly represented by either a planar diode or a two-concentricsphere diode, attempts to use the voltage wave-forms $V(t)$ calculated for such geometries resulted in a rather small decrease of the transient oscillation. Figure 8 compares the voltage vave-forms numerically-calculated with the wave-forms obtained for the planar and spherical diodes.

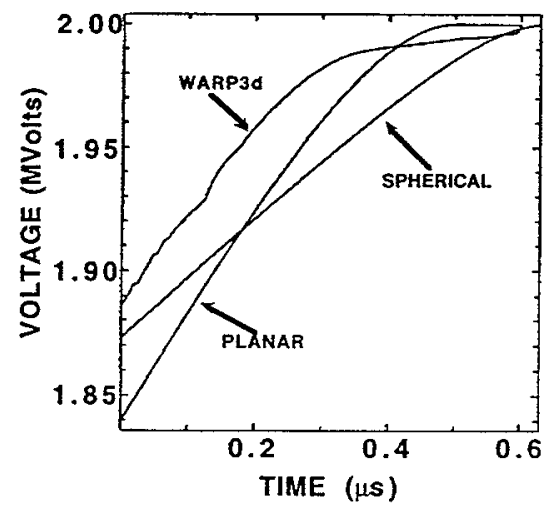

Figure 8: Voltage wave-form as calculated and used in WARP3d, and the corresponding ideal wave-forms for the planar- and spherical-equivalent diodes.

\section{CONCLUSION}

A generalization, to any complex geometry, of a method proposed by Lampel and Tiefenback to eliminate transient oscillations in a one-dimensional planar diode has been shown to give good results when implemented numerically in the three-dimensional PIC code WARP3d.

\section{REFERENCES}

[1] W.B. Herrmannsfeldt, "EGUN- An electron optics and gun design program," SLAC-Report-331. 1988.

[2] D.W. Hewett and D.J. Larsen, "The Best of Gymnos: a user's guide", UCRL-ID-110499, May 1992.

[3] A. Friedman, D. Grote and I. Haber, "Threedimensional particle simulation of heavy-ion fusion beams," Phys. Fluids B4. 2203(1992).

[4] M. Lampel and M. Tiefenback, "Elimination of current transients in a one-dimensional heavy-ion diode", LBL-14416, 1983.

[5] A. Kadish, W. Peter and M.E. Jones, "A Generalization of the Child-Langmuir Relation for OneDimensional Time-Dependent Diodes", LA-10207-MS. 1984.

[6] M. Tiefenback, M. Lampel and A. Faltens, "Optimization of Current Pulse Shapes in a One Dimensional Diode", HIFAN-Note 179, 1982.

[7] J.J Kung, "Current Transient Elimination in a One-Dimensional Diode", B.S. Thesis, University of California, Berkeley, HIFAR-Note 344. 1992.

[8] C.A. Friesen, "Modeling the Transients of a Spherical Diode", B.A. Thesis, University of California, Santa Cruz, HIFAR-Note 400, 1993. 